\title{
Detection of SPM and IMP metallo- $\beta$-lactamases in clinical specimens of Pseudomonas aeruginosa from a Brazilian public tertiary hospital
}

\author{
Authors \\ Carlos Henrique \\ Camargo $^{1}$ \\ Ariane Bruder- \\ Nascimento ${ }^{2}$ \\ Alessandro Lia Mondelli ${ }^{3}$ \\ Augusto Cezar Montelli ${ }^{4}$ \\ Terue Sadatsune ${ }^{3}$ \\ ${ }^{1}$ Biologist; MSc Student, \\ Universidade Estatual \\ Paulista (UNESP), \\ Botucatu, SP, Brazil \\ ${ }^{2} \mathrm{MSc}$; PhD Student, \\ UNESP, Botucatu, SP, Brazil \\ ${ }^{3} \mathrm{PhD}$; Professors, UNESP, \\ Botucatu, SP, Brazil \\ ${ }^{4}$ Post-doc; Professor, \\ UNESP, Botucatu, SP, Brazil
}

Submitted on: $07 / 21 / 2010$ Approved on: 12/12/2010

\section{Correspondence to: \\ Carlos Henrique Camargo Universidade Estadual Paulista \\ Instituto de Biociências de Botucatu \\ Departamento de \\ Microbiologia e \\ Imunologia \\ Distrito de Rubião Jr., s/n, Rubião Jr., \\ 18618-000, Botucatu, SP, Brazil \\ chcamargo@fmb.unesp.br}

We declare no conflict of interest.

(c)2011 Elsevier Editora Ltda. All rights reserved.

\begin{abstract}
Phenotypic and genotypic SPM and IMP metallo- $\beta$-lactamases (MBL) detection and also the determination of minimal inhibitory concentrations (MIC) to imipenem, meropenem and ceftazidime were evaluated in 47 multidrug-resistant Pseudomonas aeruginosa isolates from clinical specimens. Polymerase chain reaction detected 14 positive samples to either $b l a_{\mathrm{SPM}}$ or $b l a_{\mathrm{IMP}}$ genes, while the best phenotypic assay (ceftazidime substrate and mercaptopropionic acid inhibitor) detected 13 of these samples. Imipenem, meropenem and ceftazidime MICs were higher for MBL positive compared to MBL negative isolates. We describe here the SPM and IMP MBL findings in clinical specimens of $P$. aeruginosa from the University Hospital of Botucatu Medical School, São Paulo, Brazil, that reinforce local studies showing the high spreading of $b l a_{\mathrm{SPM}}$ and $b l a_{\mathrm{IMP}}$ genes among Brazilian clinical isolates.
\end{abstract}

Keywords: Pseudomonas aeruginosa; drug resistance, bacterial; carbapenems; metalloproteins; polymerase chain reaction.

\section{INTRODUCTION}

The most common cause of Gram-negative bacterial resistance to $\beta$-lactam antimicrobial agents is the $\beta$-lactamases production, which is highly diversified and spread in several bacteria. ${ }^{1,2}$ Metallo- $\beta$-lactamase (MBL) belongs to Group 3 of enzymes proposed by Bush and Jacoby, ${ }^{2}$ and shows ability to hydrolyze carbapenems instead of cephalosporins, cephamycins, and penicillins. Contrasting to serine- $\beta$-lactamases, MBL has poor ability to hydrolyze monobactams. ${ }^{2}$ Pseudomonas aeruginosa is one of the most common causes of nosocomial infections, persisting at hospital environment and acquiring mobile elements of resistance. ${ }^{3}$ The emergency of MBL in clinical isolates of $P$. aeruginosa is worrisome, due to the reduction of therapeutic options and the spreading ability of this bacterium. In addition, longer length of hospital stay and high mortality rates are associated to MBL producing $P$. aeruginosa infections. ${ }^{3}$ Besides $\beta$-lactam resistance, $P$. aeruginosa susceptible only to colistin or polymyxin B has been described ${ }^{3-5}$ in several infectious processes, which demand the increase use of these drugs. An accurate, easy-to-perform and fast phenotypic test to detect MBL is important to implement infection control measures, and to apply the most appropriate therapy. ${ }^{6,7}$ Several studies analyzed different substrata and MBL inhibitors associations researching MBL-producing bacteria, ${ }^{7,8}$ but no standardization to MBL detection has been published so far by guidelines, namely Clinical and Laboratory Standards Institute (CLSI). E-test strips have also been employed to detect MBL enzymes. ${ }^{8}$ In Brazil, São Paulo metallo- $\beta$-lactamase (SPM) and imipenemase (IMP) are prevalent in $P$. aerugino$s a .{ }^{7,9,10}$ In the present study, we aimed to detect MBL production in clinical specimens of $P$. aeruginosa isolated from patients of a Brazilian public tertiary hospital, and providing data to epidemiology of MBL in Brazil.

Forty-seven $P$. aeruginosa strains isolated between 2006 and 2007 were evaluated. The isolates were recovered from clinical specimens collected from patients attended at University Hospital of Botucatu Medical School, São Paulo State, Brazil, a regional reference tertiary hospital. Isolates were chosen due to their multidrugresistant profile, according to disk-diffusion susceptibility test and CLSI cutoffs, ${ }^{11}$ and the sole susceptibility to polymyxin B, confirmed by E-test (AB Biodisk) strips [minimal inhibitory 
concentrations (MIC) ranging from 0.094 to $2 ; \mathrm{MIC}_{50}$ : 0.5 ; MIC $_{90}: 1.5 \mu \mathrm{g} / \mathrm{mL} ; 100 \%$ susceptible]. MICs were determined (in duplicate) by microdilution broth method for imipenem (ABL), meropenem (Astra Zeneca) and ceftazidime (NovaFarma); quality controls were performed with Escherichia coli ATCC 25922 and P. aeruginosa ATCC 27853 strains. MBL phenotypic detection was evaluated by the double-disk synergy test (DDST) with imipenem or ceftazidime disks (Oxoid) as substrata, and mercaptopropionic acid (Sigma-Aldrich), mercaptoacetic acid (Sigma-Aldrich), or ethylenediamine tetra-acetic acid (EDTA, Sigma-Aldrich) as MBL inhibitors, according to Picão et al. ${ }^{7}$ Genotypic detection targeting to the $b l a_{\mathrm{SPM}}$ and $b l a_{\mathrm{IMP}}$ genes was carried out by polymerase chain reaction (PCR), according to protocols published elsewhere. ${ }^{9}$ Positive and negative quality control strains were included to assess the quality of phenotypic and genotypic assays. Student's $t$ test was carried out to compare the median MIC of positive and negative MBL strains; sensitivity and specificity were calculated for the different substrata antimicrobials and MBL inhibitors assessed in the DDST; p-values below 0.05 were considered statistically significant. The study was approved by the local Ethics Committee (Protocol 2886-2008).

Forty-seven isolates of $P$. aeruginosa were analyzed, from urine (17), blood (5), and various other specimens (25). The distribution of isolates across the hospital was diversified, being, even in low numbers, mostly isolated from Emergency Unit and Urology Wards (5 each), and Central Intensive Care Unit (4). MIC values range (and MIC that inhibited the growth of $90 \%$ of the strains, $\mathrm{MIC}_{90}$ ) for imipenem, meropenem and ceftazidime, were, respectively, 16 to 512 (512); 16 to $>512$ (512); 4 to $>512$ $(512 \mu \mathrm{g} / \mathrm{mL})$. MIC median for MBL-positive strains were significantly higher than those MIC for MBL-negative strains ( $p$ 0.0001), for each drug: imipenem, meropenem and ceftazidime (Figure 1). Polymyxin B MIC median also showed difference between MBL-positive and -negative strains ( 0.98 vs. $0.66 \mu \mathrm{g} / \mathrm{mL}$, respectively; $\mathrm{p}=0.0097$ ). $\mathrm{MBL}$ genotypic detection was carried out with specific primers for $b l a_{\mathrm{SPM}}$ and $b l a_{\mathrm{IMP}}$ genes. Fourteen (29.8\%) isolates in 47 samples were either $b l a_{\mathrm{SPM}}$ or $b l a_{\mathrm{IMP}}$ positive. $b l a_{\mathrm{SPM}}$ gene accounted for $71 \%(10 / 14)$ positive MBL strains, while $b l a_{\mathrm{IMP}}$ was detected in $29 \%$ (4/14). DDST employing mercaptopropionic acid and ceftazidime showed the best association to PCR results, with sensitivity of $92.8 \%$ and specificity of $100 \%$. MBL-positive $P$. aeruginosa strains were isolated from different clinical sources (urine, blood, and other biological fluids) and from several wards: Gastric Surgery, Gastroenterology, Intensive Care Unit, Intern Medicine, Orthopedics, Urology and Agreement Ward.

$P$. aeruginosa is an important nosocomial agent, and several resistance mechanisms have been related on this microorganism; ${ }^{12,13} \mathrm{MBL}$ has been emerging as a worrying resistance mechanism in this and in several other Gram-

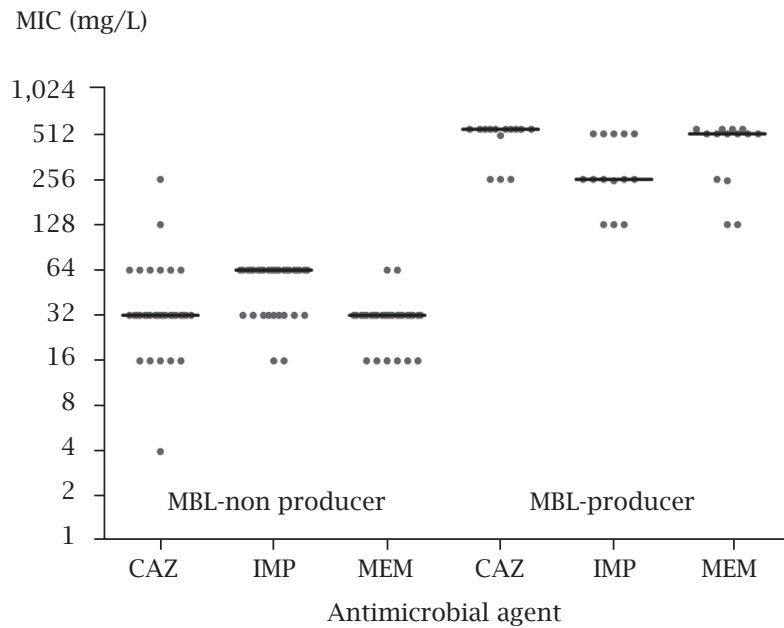

Figure 1: Comparison between MIC of MBL-negative and positive- $P$. aeruginosa strains. o represents each isolate; horizontal bars represent average values. CAZ, ceftazidime; IMP, imipenem; MEM, meropenem.

negative rods. ${ }^{2} \beta$-lactamases are currently classified by two ways: based on the amino acid sequence (molecular classification) or based on the hydrolytic and inhibition properties (functional classification). ${ }^{2}$ According to molecular classification $\beta$-lactamases are grouped into class $\mathrm{A}, \mathrm{B}, \mathrm{C}$ and $\mathrm{D}$. While class $\mathrm{A}, \mathrm{C}$ and $\mathrm{D}$ enzymes are characterized to utilize serine for $\beta$-lactam hydrolysis, class $B$ enzymes are metalloenzymes, that use divalent cations as cofactors to substrate hydrolysis. ${ }^{2}$ Functional classification divides $\beta$-lactamases enzymes into 3 groups: group 1, cephalosporinases; group 2 , the largest one, which contains enzymes that hydrolyses penicillins, cephalosporins, monobactams, carbenicillin, cloxacillin, and carbapenems; and group 3, metallo-carbapenemase. Colistin-only susceptible $P$. aeruginosa is a relatively contemporary issue, once carbapenems remained as effective therapeutic choice until the carbapenemases emergence on the last two decades. ${ }^{7,8,14}$ Investigating MBL-encoding genes, we found isolates carrying $b l a_{\mathrm{SPM}}$ and $b a_{\mathrm{IMP}}$ genes that together correspond to $29.8 \%$ of evaluated samples. In Brazil, the dissemination of $P$. aeruginosa carrying $b l a_{\mathrm{SPM}}$ gene has been reported throughout from several regions, ${ }^{15,16}$ and SPM is the prevalent MBL among the isolates, although other enzymes have also been described, but with low frequency. ${ }^{9,10,16,17}$ SPM carbapenemase was the main mechanism that conferred resistance to ceftazidime on ceftazidime-resistant P. aeruginosa isolated from bloodstream infections. ${ }^{10} \mathrm{MBL}$ rate found here is higher than that one reported by Viana Vieira et al..$^{14}$ (7.5\%), but it is lower than those published by other Brazilian researchers (35.9 to $80.4 \%$ ). ${ }^{9-11,18-20}$ Zavascki et al. ${ }^{3}$ found $30 \mathrm{P}$. aeruginosa only susceptible to polymyxin $\mathrm{B}$ among $86 \mathrm{MBL}$ producing strains and the $b l a_{\mathrm{SPM}}$ gene was detected in all the 14 strains randomly selected, and 
Gräf et al ${ }^{19}$ also detected the $b l a_{\text {SPM }}$ gene in strains only susceptible to polymyxin B, both from Brazilian hospitals. Our data are in accordance to these studies, showing that SPM is the prevalent MBL among Brazilian P. aeruginosa isolates. The lower frequency of MBL in our patient population might be the result of our inclusion criteria (only susceptibility to polymyxin B), once that resistance to imipenem and meropenem simultaneously suggests the evolvement of carbapenem resistance mechanism other than the enzymatic one, such as porin loss and/or overexpression of efflux pumps. ${ }^{10}$ High MIC values for carbapenems and ceftazidime are associated to MBL production, and this may be a useful tool to differentiate from other resistance mechanism, such as efflux pumps or chromosomal inducible AmpC $\beta$-lactamase. ${ }^{1}$ Recent studies confirm this tendency, ${ }^{5,9,10,12}$ and our data also showed this remark (Figure 1). Some peculiar serine- $\beta$-lactamases, such as GES-5, can also present hydrolytic activity against carbapenems in $P$. aeruginosa isolates; this activity is, however, lower than that one presented by MBL-encoding genes isolates, as recently reported in a Brazilian study. ${ }^{10}$ Resistance to other antimicrobial agent classes ${ }^{12,13}$ reduces the therapeutic options to manage multidrug-resistant $P$. aeruginosa clinical isolates, and it seems to be even more recurrent to employ last resource drugs, such as polymyxins, to treat these infections. Polymyxin B showed efficacy against all of the isolates here evaluated. Similar observation was reported by Lee et al. ${ }^{5}$ who studied 17 colistin-only sensitive isolates from Korea (MIC ranging from 0.5-2.0 $\mu \mathrm{g} / \mathrm{mL}$ ). In imipenem-resistant $P$. aeruginosa strains isolated from a Brazilian tertiary care university hospital, polymyxin B showed great activity (100\% susceptible; $\mathrm{MIC}_{90} \leq 0.5 \mathrm{mgL}$ ). ${ }^{9}$ However, we can wonder how much longer these bacteria will persist susceptible to these remaining drugs if this selective pressure goes on. Comparing different phenotypic tests to detect MBL, we found that ceftazidime disk and mercaptopropionic acid association, in a double disk synergy test, was the most sensitive and specific assay, as it was reported by Picão et al. ${ }^{7}$ We observed that all other combinations of substrata and inhibitors (imipenem and mercaptopropionic acid; imipenem and mercaptoacetic acid; imipenem and EDTA; ceftazidime and mercaptoacetic acid; ceftazidime and EDTA) showed maximum specificities (100\%), but low sensitivity to these combinations (zero to $71.4 \%$ ), restricting their utilization. In spite of the high cost, MBL E-test strips can be employed to detect the MBL-producing $P$. aeruginosa due its high sensibility and specificity. ${ }^{8}$ Despite the recommendation for screening and confirming carbapenemase production in Enterobacteriaceae, CLSI document M100-S20 ${ }^{11}$ made no mention of detecting this enzyme on non-fermentative Gram-negative bacteria. Early identification of MBL in clinical specimens is important to provide correct antimicrobial therapy guidance, once appropriate therapy implementation can nullify the worst prognostic of MBL producing
P. aeruginosa. ${ }^{3}$ When detected, it is possible to avoid the dissemination of such strains among patients ${ }^{7-9}$ and to avoid the MBL genetic determinants spread to different species of Gram-negative bacteria, as Enterobacteriaceae. ${ }^{6}$ The contention of $\mathrm{MBL}$ producing $P$. aeruginosa is highly recommended, because patient-to-patient transmission may lead these strains to be endemic in such institution ${ }^{3}$ or among different hospitals. ${ }^{15-17}$ In Brazil, Gales et al. ${ }^{16}$ reported the spreading of $b l a_{\mathrm{SPM}^{-}}$ producing $P$. aeruginosa clone throughout the country. The non-centralized distribution of positive $\mathrm{MBL}$ P. aeruginosa from Hospital of Botucatu Medical School (i.e., the recovering of MBL $P$. aeruginosa from hospitalized patients from several wards) suggests the non-clonal spreading of $P$. aeruginosa strains, although further molecular analyses are necessary to confirm this hypothesis. Our results contribute to the study of MBL epidemiology in Brazil, mainly to $b l a_{\mathrm{SPM}}$ spreading. It is worthwhile to emphasize that, after restricted by more than ten years to Brazilian hospitals, SPM seems to become a global challenge, ${ }^{15,20}$ warning for the role of human traffic in spreading MBL genes. ${ }^{6,20}$

\section{ACKNOWLEDGEMENTS}

The authors thank to D. O. Garcia and to A. C. C. Pignatari, whose, respectively, kindly provided $b l a_{\mathrm{SPM}}$ and $b l a_{\mathrm{IMP}}$ positive controls; J. Rodrigues, C. M. Thomazini, and F. P. C. Pedrosa, for providing technical support in PCR techniques, and the staff of the Microbiological Laboratory from the University Hospital of Botucatu Medical School.

\section{REFERENCES}

1. Livermore D. Beta-Lactamases in laboratory and clinical resistance. Clin Microbiol Rev 1995; 8:557-84.

2. Bush K, Jacoby G. Updated functional classification of beta-lactamases. Antimicrob Agents Chemother 2010; 54:969-76.

3. Zavascki A, Barth A, Gonçalves A et al. The influence of metallo-beta-lactamase production on mortality in nosocomial Pseudomonas aeruginosa infections. J Antimicrob Chemother 2006; 58:387-92.

4. Mastoraki A, Douka E, Kriaras I et al. Pseudomonas aeruginosa susceptible only to colistin in intensive care unit patients. Surg Infect (Larchmt) 2008; 9:153-60.

5. Lee Y, Ahn B, Jin J et al. Molecular characterization of Pseudomonas aeruginosa isolates resistant to all antimicrobial agents, but susceptible to colistin, in Daegu, Korea. J Microbiol 2007; 45:358-63.

6. Walsh T, Toleman M, Poirel L et al. Metallo-beta-lactamases: the quiet before the storm? Clin Microbiol Rev 2005; 18:306-25.

7. Picão R, Andrade S, Nicoletti A et al. Metallo-beta-lactamase detection: comparative evaluation of double-disk synergy versus combined disk tests for IMP-, GIM-, SIM-, SPM-, or VIM-producing isolates. J Clin Microbiol 2008; 46:2028-37. 
8. Pitout J, Gregson D, Poirel L et al. Detection of Pseudomonas aeruginosa producing metallo-beta-lactamases in a large centralized laboratory. J Clin Microbiol 2005; 43:3129-35.

9. Sader H, Reis A, Silbert S, Gales A. IMPs, VIMs and SPMs: the diversity of metallo-beta-lactamases produced by carbapenemresistant Pseudomonas aeruginosa in a Brazilian hospital. Clin Microbiol Infect 2005; 11:73-6.

10. Picão R, Poirel L, Gales A et al. Diversity of beta-lactamases produced by ceftazidime-resistant Pseudomonas aeruginosa isolates causing bloodstream infections in Brazil. Antimicrob Agents Chemother 2009; 53:3908-13.

11. Clinical and Laboratory Standards Institute. Performance standards for antimicrobial susceptibility testing; 20th informational supplement. M100-S20. Wayne, PA: Clinical and Laboratory Standards Institute; 2010.

12. Doi Y, Ghilardi A, Adams J et al. High prevalence of metallobeta-lactamase and 16S rRNA methylase coproduction among imipenem-resistant Pseudomonas aeruginosa isolates in Brazil. Antimicrob Agents Chemother 2007; 51:3388-90.

13. Bonomo R, Szabo D. Mechanisms of multidrug resistance in Acinetobacter species and Pseudomonas aeruginosa. Clin Infect Dis 2006; 43(Suppl 2):S49-56.

14. Viana Vieira V, Lourenço da Fonseca E, Paulo Vincente A. Metallo-beta-lactamases produced by carbapenem-resistant Pseudomonas aeruginosa in Brazil. Clin Microbiol Infect 2005; 11:937.
15. Fonseca E, Freitas F, Vicente A. The colistin-only-sensitive (COS) Brazilian Pseudomonas aeruginosa clone SP (ST $277)$ is spread worldwide. Antimicrob Agents Chemother 2010; 54: 2743.

16. Gales A, Menezes L, Silbert S et al. Dissemination in distinct Brazilian regions of an epidemic carbapenemresistant Pseudomonas aeruginosa producing SPM metallo-beta-lactamase. J Antimicrob Chemother 2003; 52:699702 .

17. Martins A, Zavascki A, Gaspareto P et al. Dissemination of Pseudomonas aeruginosa producing SPM-1-like and IMP1-like metallo-beta-lactamases in hospitals from southern Brazil. Infection 2007; 35:457-60.

18. Poirel L, Magalhaes M, Lopes M et al. Molecular analysis of metallo-beta-lactamase gene bla (SPM-1)-surrounding sequences from disseminated Pseudomonas aeruginosa isolates in Recife, Brazil. Antimicrob Agents Chemother 2004; 48:1406-9.

19. Gräf T, Fuentefria D, Corção G. Occurrence of multiresistant strains of Pseudomonas aeruginosa producing metallo-beta-lactamase blaSPM-1 in clinical samples. Rev Soc Bras Med Trop 2008; 41:306-8.

20. Salabi A, Toleman M, Weeks J et al. First report of the metallo-beta-lactamase SPM-1 in Europe. Antimicrob Agents Chemother 2010; 54:582. 